\title{
On Some Properties of Leibniz's Triangle
}

\author{
R. Sivaraman \\ Department of Mathematics, D. G. Vaishnav College, Chennai, India
}

Received February 17, 2021; Revised March 11, 2021; Accepted April 22, 2021

\section{Cite This Paper in the following Citation Styles}

(a): [1] R. Sivaraman, "On Some Properties of Leibniz's Triangle," Mathematics and Statistics, Vol. 9, No. 3, pp. 209 217, 2021. DOI: 10.13189/ms.2021.090301.

(b): R. Sivaraman (2021). On Some Properties of Leibniz's Triangle. Mathematics and Statistics, 9(3), 209 - 217. DOI: 10.13189/ms.2021.090301.

Copyright@2021 by authors, all rights reserved. Authors agree that this article remains permanently open access under the terms of the Creative Commons Attribution License 4.0 International License

\begin{abstract}
One of the Greatest mathematicians of all time, Gotfried Leibniz, introduced amusing triangular array of numbers called Leibniz's Harmonic triangle similar to that of Pascal's triangle but with different properties. I had introduced entries of Leibniz's triangle through Beta Integrals. In this paper, I have proved that the Beta Integral assumption is exactly same as that of entries obtained through Pascal's triangle. The Beta Integral formulation leads us to establish several significant properties related to Leibniz's triangle in quite elegant way. I have shown that the sum of alternating terms in any row of Leibniz's triangle is either zero or a Harmonic number. A separate section is devoted in this paper to prove interesting results regarding centralized Leibniz's triangle numbers including obtaining a closed expression, the asymptotic behavior of successive centralized Leibniz's triangle numbers, connection between centralized Leibniz's triangle numbers and Catalan numbers as well as centralized binomial coefficients, convergence of series whose terms are centralized Leibniz's triangle numbers. All the results discussed in this section are new and proved for the first time. Finally, I have proved two exceedingly important theorems namely Infinite Hockey Stick theorem and Infinite Triangle Sum theorem. Though these two theorems were known in literature, the way of proving them using Beta Integral formulation is quite new and makes the proof short and elegant. Thus, by simple re-formulation of entries of Leibniz's triangle through Beta Integrals, I have proved existing as well as new theorems in much compact way. These ideas will throw a new light upon understanding the fabulous Leibniz's number triangle.
\end{abstract}

Keywords Leibniz's Triangle, Pascal's Triangle, Harmonic Numbers, Centralized Leibniz's Triangle numbers, Inverted Infinite Hockey Stick Property, Infinite Triangle Sum Property

\section{Introduction}

The great German polymath Gotfried Wilhelm Leibniz, who was one of the discoverers of Calculus introduced a number triangle containing unit fractions which satisfy certain recurrence relation condition. Leibniz's triangle described by Leibniz in 1673 contains several interesting properties which were explored by many later mathematicians. During the time Leibniz introduced his harmonic triangle, another mathematician Pietro Mengoli discussed the same version of the triangle for finding quadratures(areas) of a semi-circles with different diameters. But Leibniz defined the elements of the triangle recursively and so the triangle got the name Leibniz's triangle. We may also call Leibniz's triangle as Harmonic Triangle as the outer diagonal of Leibniz's triangle are occupied by Harmonic numbers. In this paper, I will introduce the elements of Leibniz's triangle in a different perspective through Beta Integrals and discuss several properties through this new definition.

\section{Definitions}

\subsection{The Beta Function denoted by $B(m, n)$ where} $m, n>0$ is Defined by the Integral

$$
B(m, n)=\int_{0}^{1} x^{m-1}(1-x)^{n-1} d x \quad(2.1) \text {. We may also call }
$$


$B(m, n)$ as Beta Integral.

By considering $f(x)=x^{m-1}(1-x)^{n-1}$ we see that $f(1-x)=x^{n-1}(1-x)^{m-1}$

By the property of the definite integrals $\int_{0}^{1} f(x) d x=\int_{0}^{1} f(1-x) d x(2.2)$ we see that

$B(m, n)=\int_{0}^{1} x^{m-1}(1-x)^{n-1} d x=\int_{0}^{1} x^{n-1}(1-x)^{m-1} d x=B(n, m)$

Hence, $B(m, n)=B(n, m)$ (2.3). Thus the Beta Integral defined in (2.1) is symmetric with respect to the indices $m$ and $n$.

\section{Construction of Leibniz's Triangle}

The Pascal's triangle consists of entries which are binomial coefficients of the form $P(m, n)=\left(\begin{array}{l}m \\ n\end{array}\right)$ where $0 \leq n \leq m$. The Pascal's triangle is displayed in Figure 1.

$$
\begin{aligned}
& 1 \\
& 11 \\
& \begin{array}{lll}
1 & 2 & 1
\end{array} \\
& \begin{array}{llll}
1 & 3 & 3 & 1
\end{array} \\
& \begin{array}{lllllll}
1 & 4 & 6 & 4 & 1
\end{array} \\
& \begin{array}{lllllll}
1 & 6 & 15 & 20 & 15 & 6 & 1
\end{array} \\
& \begin{array}{llllllll}
1 & 7 & 21 & 35 & 35 & 21 & 7 & 1
\end{array} \\
& \begin{array}{lllllllll}
1 & 8 & 28 & 56 & 70 & 56 & 28 & 8 & 1
\end{array} \\
& \begin{array}{llllllllll}
1 & 9 & 36 & 84 & 126 & 126 & 84 & 36 & 9 & 1
\end{array} \\
& \begin{array}{lllllllllll}
1 & 10 & 45 & 120 & 210 & 252 & 210 & 120 & 45 & 10 & 1
\end{array}
\end{aligned}
$$

Figure 1. The first 11 rows of Pascal's Triangle

Pascal's Triangle possesses so many exciting properties that a whole book can be devoted to listing them. Now to construct Leibniz's triangle from Pascal's triangle in Figure 1, we need to take the reciprocal of each number in row $m$, and multiply them by $\frac{1}{m+1}$.

For example, the entry corresponding to $m=0$ in Leibniz triangle would be $\frac{1}{1} \times \frac{1}{1}=1$.

Similarly the entries corresponding to $m=1$ in Leibniz triangle are $\frac{1}{2} \times \frac{1}{1}=\frac{1}{2}, \frac{1}{2} \times \frac{1}{1}=\frac{1}{2}$

The entries corresponding to $m=2$ in Leibniz triangle are $\frac{1}{3} \times \frac{1}{1}=\frac{1}{3}, \frac{1}{3} \times \frac{1}{2}=\frac{1}{6}, \frac{1}{3} \times \frac{1}{1}=\frac{1}{3}$

The entries corresponding to $m=3$ in Leibniz triangle are

$$
\frac{1}{4} \times \frac{1}{1}=\frac{1}{4}, \frac{1}{4} \times \frac{1}{3}=\frac{1}{12}, \frac{1}{4} \times \frac{1}{3}=\frac{1}{12}, \frac{1}{4} \times \frac{1}{1}=\frac{1}{4}
$$

Proceeding in this way for each value of $m$, we get Leibniz's triangle as shown in Figure 2, from the Pascal's triangle of Figure 1.

Sometimes, we call the Leibniz's triangle in Figure 2 as Harmonic triangle, since the outer diagonal entries are Harmonic numbers $1, \frac{1}{2}, \frac{1}{3}, \frac{1}{4}, \frac{1}{5}, \frac{1}{6}, \ldots$ Further, we note that the $n$th entry in row $m$ of Leibniz's triangle where $0 \leq n \leq m$ shown in Figure 2 is given by $P L(m, n)=\frac{1}{m+1} \times \frac{1}{\left(\begin{array}{l}m \\ n\end{array}\right)}=\frac{(m-n) ! \times n !}{(m+1) !}$ where

$P L(m, n)$ is the Leibniz's triangle entry obtained from corresponding Pascal's triangle entry. For knowing more about Leibniz and his fantastic number triangle refer [1 $10]$.

Leibniz's triangle exhibit many exciting properties similar to that of Pascal's triangle. The upcoming sections focus on exhibiting such properties. For doing this, I will introduce entries of Leibniz's triangle in a completely different way.

$$
\begin{aligned}
& 1
\end{aligned}
$$

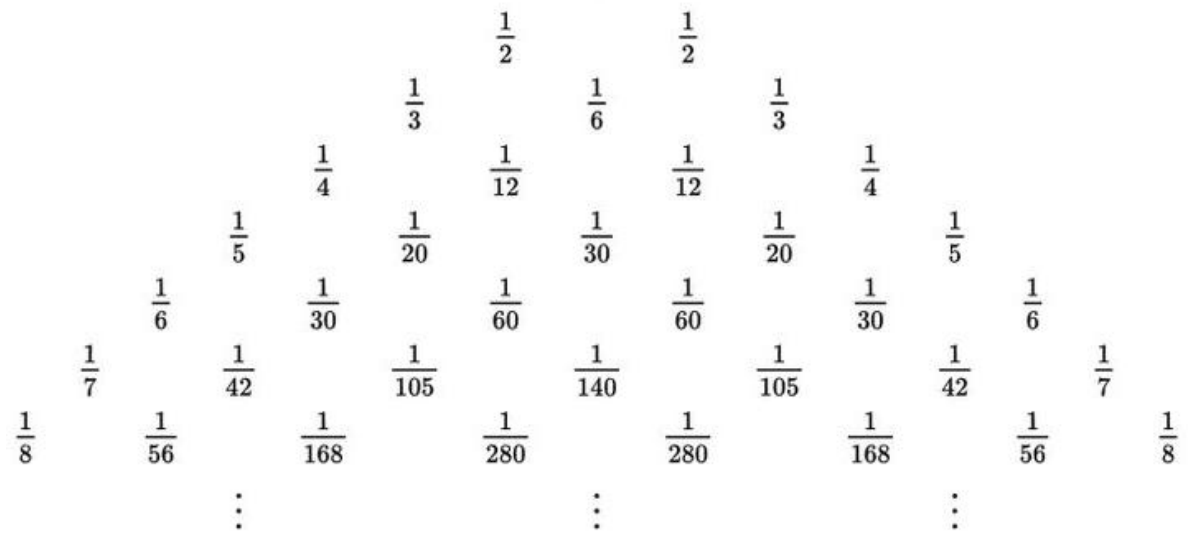

Figure 2. The first 8 rows of Leibniz's Triangle 


\section{New Formulation of Leibniz's Triangle}

4.1. Let $m, n \geq 0$ be integers. We denote $L(m, n)$ to be the entries of Leibniz's triangle as shown in Figure 3

$$
\begin{aligned}
& L_{0,0} \\
& L_{1,0} \quad L_{0,1} \\
& L_{2,0} \quad L_{1,1} \quad L_{0,2} \\
& \begin{array}{llll}
L_{3,0} & L_{2,1} & L_{1,2} & L_{0,3}
\end{array} \\
& \begin{array}{lllll}
L_{4,0} & L_{3,1} \quad L_{2,2} \quad L_{1,3} & L_{0,4}
\end{array}
\end{aligned}
$$

Figure 3. Arrangement of entries in Leibniz's Triangle

\subsection{The Entries $L_{m, n}$ of Leibniz's Triangle in Figure 3 is}

Defined by the Integral $L_{m, n}=\int_{0}^{1} x^{m}(1-x)^{n} d x$

In view of (2.1), we can write (4.1) as $L_{m, n}=B(m+1, n+1)(4.2)$

Using the relationship between Beta and Gamma Integrals and noting that $\Gamma(k+1)=k$ ! for all whole numbers $k$, we have

$B(p, q)=\int_{0}^{1} x^{p-1}(1-x)^{q-1} d x=\frac{\Gamma(p) \times \Gamma(q)}{\Gamma(p+q)}=\frac{(p-1) ! \times(q-1) !}{(p+q-1) !}$

\subsection{Lemma 1}

If $m, n \geq 0$ be integers are such that $0 \leq n \leq m$ then $P L(m, n)=L_{m-n, n}$

Proof: From (3.1), (4.1), (4.2) and (4.3), we find that

$$
\begin{aligned}
& L_{m-n, n}=\int_{0}^{1} x^{m-n}(1-x)^{n} d x \\
& =B(m-n+1, n+1) \quad \text { as required. } \\
& =\frac{(m-n) ! \times n !}{(m+1) !}=P L(m, n)
\end{aligned}
$$

Lemma 1, thus establishes the fact that the entries of Leibniz's triangle obtained through Pascal's triangle are exactly the same as entries of the reformulation as defined in (4.1).

\section{Properties of Leibniz's Triangle}

In view of (4.2), having identified the entries of Leibniz's triangle as the Beta integral, in this section I prove the following interesting and basic properties concerning Leibniz's triangle.

\subsection{Symmetric Property}

The entries of Leibniz's triangle are symmetric with respect to vertical line through the centre i.e. $L_{m, n}=L_{n, m}$ (5.1)

Proof: By (2.3) we know that Beta Integral is symmetric with respect to its indices $m$ and $n$. Hence, from (4.2), we get $L_{m, n}=B(m+1, n+1)=B(n+1, m+1)=L_{n, m}$ proving

\subsection{Harmonic Numbers Property}

The outermost diagonal entries in the Leibniz's triangle are Harmonic numbers of the form $\frac{1}{k+1}, k=0,1,2,3,4, \ldots$

Proof: The outermost diagonals in the Leibniz's triangle (by Figure 3) are numbers of the form $L_{k, 0}$ or $L_{0, k}$ where $k=0,1,2,3,4, \ldots$ But by (5.1), we find that $L_{k, 0}=L_{0, k}$ for all $k$.

From (4.1), we have $L_{m, n}=\int_{0}^{1} x^{m}(1-x)^{n} d x$. Hence, $L_{k, 0}=\int_{0}^{1} x^{k} d x=\frac{1}{k+1}(5.2)$. But we know that $L_{k, 0}=L_{0, k}$. Hence from (5.2), we see that the outmost diagonal entries of the Leibniz's triangle are Harmonic numbers of the form $\frac{1}{k+1}, k=0,1,2,3,4, \ldots$

\subsection{Recurrence Relation}

The entries of the Leibniz's triangle satisfy the recurrence relation given by $L_{m-1, n+1}=L_{m-1, n}-L_{m, n}$

$$
\begin{aligned}
& \text { Proof: } \quad \begin{array}{l}
\text { Using } \\
L_{m-1, n+1}+L_{m, n}=\int_{0}^{1} x^{m-1}(1-x)^{n+1} d x+\int_{0}^{1} x^{m}(1-x)^{n} d x \\
=\int_{0}^{1} x^{m-1}(1-x)^{n}[(1-x)+x] d x=\int_{0}^{1} x^{m-1}(1-x)^{n} d x=L_{m-1, n}
\end{array}
\end{aligned}
$$

This provides (5.3) and completes the proof.

Many sources about Leibniz's triangle use the recurrence relation property in (5.3) to generate subsequent entries of Leibniz's triangle.

\subsection{Infinite Sum Property}

The sum of reciprocals of all triangular numbers is 2, i.e. $\sum_{m=1}^{\infty} \frac{2}{m(m+1)}=2$

Proof: In Figure 3 of Leibniz's triangle we consider the second leading diagonal of the form $L_{m, 1}$ where $m=0,1,2,3,4, \ldots$ 
Now from (4.1), we have

$L_{m, 1}=\int_{0}^{1} x^{m}(1-x) d x=\frac{1}{m+1}-\frac{1}{m+2}=\frac{1}{(m+1)(m+2)}$

Triangular numbers are numbers of the form $\frac{m(m+1)}{2}, m=1,2,3,4, \ldots$

Hence the sum of reciprocals of all triangular numbers $=$ $\sum_{m=1}^{\infty} \frac{2}{m(m+1)}=2 \sum_{m=0}^{\infty} \frac{1}{(m+1)(m+2)}$

Now from (5.5), we get

Sum of reciprocals of all triangular numbers = $2 \sum_{m=0}^{\infty} L_{m, 1}=2 \sum_{m=0}^{\infty} \int_{0}^{1} x^{m}(1-x) d x$

$$
=2 \int_{0}^{1}\left(\sum_{m=0}^{\infty} x^{m}\right)(1-x) d x=2 \int_{0}^{1} \frac{1}{1-x}(1-x) d x=2 .
$$

This completes the proof.

\subsection{Sum of Alternating Terms}

The sum of alternating terms in $m$ th row of the Leibniz's triangle is zero, if $m$ is odd and a Harmonic number if $m$ is even (5.6)

Proof: From Figure 3, we first notice that the sum of alternating terms in $m$ th row of the Leibniz's triangle = $\sum_{n=0}^{m}(-1)^{n} L_{m-n, n}$

We will now try to compute the sum given in (5.7). Using (4.1), we get

$$
\begin{aligned}
\sum_{n=0}^{m}(-1)^{n} L_{m-n, n} & =\sum_{n=0}^{m}(-1)^{n} \int_{0}^{1} x^{m-n}(1-x)^{n} d x=\int_{0}^{1} x^{m}\left(\sum_{n=0}^{m}\left(\frac{x-1}{x}\right)^{n}\right) d x \\
& =\int_{0}^{1} x^{m}\left[\frac{x^{m+1}-(x-1)^{m+1}}{x^{m}}\right] d x=\frac{1}{m+2}\left[1+(-1)^{m}\right]
\end{aligned}
$$

If $m$ is odd, then $\sum_{n=0}^{m}(-1)^{n} L_{m-n, n}=0$

If $m$ is even, then $\sum_{n=0}^{m}(-1)^{n} L_{m-n, n}=\frac{2}{m+2}$ (5.9) . In particular if $m=2 k$, where $k=0,1,2,3,4, \ldots$ then from (5.9) we get $\sum_{n=0}^{m}(-1)^{n} L_{m-n, n}=\frac{1}{k+1}, k=0,1,2,3,4, \ldots$

Note that $\frac{1}{k+1}$ for $k=0,1,2,3,4, \ldots \quad$ generates Harmonic numbers. Another occurrence of Harmonic numbers in Leibniz's triangle.

Hence, equations (5.8) and (5.10) prove (5.6). This completes the proof.

\section{Centralized Leibniz's Triangle Numbers}

We define numbers of the form $L_{m, m}$ as centralized Leibniz's triangle numbers. Since $m+m=2 m$ is always even, we find from Figure 3 that $L_{m, m}$ occurs as the middle term of the row beginning with $L_{2 m, 0}$ and ending with $L_{0,2 m}$ for $m=0,1,2,3,4, \ldots$

The Centralized Leibniz's Triangle Numbers are given by

$$
L_{m, m}=\frac{m !}{2^{m} \times(2 m+1) ! !}(6.1)
$$

where $(2 m+1) ! !=(2 m+1)(2 m-1)(2 m-3) \times \cdots \times 5 \times 3 \times 1$ is called the double factorial of $2 m+1$.

Proof: From (4.2) and (4.3), we get

$$
\begin{aligned}
& L_{m, m}=B(m+1, m+1)=\frac{m ! \times m !}{(2 m+1) !} \\
& =\frac{m ! \times m !}{(2 m+1) \times(2 m) \times(2 m-1) \times \cdots \times 4 \times 3 \times 2 \times 1} \\
& =\frac{m !}{2^{m} \times(2 m+1) ! !}
\end{aligned}
$$

This completes the proof.

If $L_{m, m}$ is the centralized Leibniz's triangle number then $L_{m, m}=4 L_{m+1, m+1} \quad(6.2)$ as $m \rightarrow \infty$

Proof: From (6.1) we have $\frac{L_{m, m}}{L_{m+1, m+1}}=\frac{\frac{m !}{2^{m} \times(2 m+1) ! !}}{\frac{(m+1) !}{2^{m+1} \times(2 m+3) ! !}}=2 \times \frac{2 m+3}{m+1} \rightarrow 4$ as $m \rightarrow \infty$.

This proves (6.2) and completes the proof.

If $C_{m}=\frac{1}{m+1}\left(\begin{array}{c}2 m \\ m\end{array}\right)$ is the $m$ th Catalan number and $L_{m, m}$ is the centralized Leibniz's triangle number then

$$
L_{m, m} \times C_{m}=\frac{1}{(m+1)(2 m+1)}
$$

Proof:

$$
\begin{aligned}
& L_{m, m} \times C_{m}=B(m+1, m+1) \times \frac{1}{m+1}\left(\begin{array}{c}
2 m \\
m
\end{array}\right) \\
& =\frac{m ! \times m !}{(2 m+1) !} \times \frac{1}{m+1} \times \frac{(2 m) !}{m ! \times m !} \\
& =\frac{1}{(m+1)(2 m+1)}
\end{aligned}
$$


This proves (6.3) and completes the proof.

The product of centralized Leibniz's triangle and corresponding centralized binomial coefficients in Pascal's triangle is the reciprocal of the odd positive integers.

Proof: Let $L_{m, m}$ be the centralized Leibniz's triangle numbers. The centralized binomial coefficients in Pascal's triangle are numbers of the form $P(2 m, m)=\left(\begin{array}{c}2 m \\ m\end{array}\right)$. We notice from the definition of Catalan numbers in 6.4 that $(m+1) C_{m}=P(2 m, m)$. Hence the product of centralized Leibniz's triangle numbers and corresponding centralized binomial coefficients, using (6.3) is given by $L_{m, m} \times P(2 m, m)=L_{m, m} \times(m+1) C_{m}=\frac{1}{2 m+1}$. Thus the required product is the reciprocal of odd positive integers for each value of $m=0,1,2,3,4, \ldots$ This completes the proof.

$$
\sum_{m=0}^{\infty} L_{m, m}=\frac{2 \pi}{3 \sqrt{3}}
$$

Proof: From (4.1), we have

$$
\begin{aligned}
\sum_{m=0}^{\infty} L_{m, m} & =\sum_{m=0}^{\infty} \int_{0}^{1} x^{m}(1-x)^{m} d x=\int_{0}^{1} \sum_{m=0}^{\infty}[x(1-x)]^{m} d x \\
& =\int_{0}^{1} \frac{1}{1-x(1-x)} d x=\int_{0}^{1} \frac{d x}{\left(x-\frac{1}{2}\right)^{2}+\left(\frac{\sqrt{3}}{2}\right)^{2}}=\frac{2 \pi}{3 \sqrt{3}}
\end{aligned}
$$

Thus the sum of centralized Leibniz's triangle numbers converges to $\frac{2 \pi}{3 \sqrt{3}}$. This proves (6.4) and completes the proof.

\section{Summations in Leibniz's Triangle}

In Pascal's triangle there is a wonderful property concerning summing entries in a particular diagonal called Hockey Stick Property. There is a similar version in Leibniz's triangle producing gallery of infinite series summations which I will prove below.

\subsection{Inverted Infinite Hockey Stick Property}

We first look at the two figures 4 and 5 displayed below. In Figures 4 and 5 we see that if we add the numbers in the infinite rectangular strip the sum would be a number indicated in the circle located at North-West direction.

Thus from Figures 4 and 5 we see that

$$
\frac{1}{2}+\frac{1}{6}+\frac{1}{12}+\frac{1}{20}+\frac{1}{30}+\frac{1}{42}+\frac{1}{56}+\cdots=1
$$

and

$$
\frac{1}{12}+\frac{1}{30}+\frac{1}{60}+\frac{1}{105}+\frac{1}{168}+\cdots=\frac{1}{6}
$$

Using (5.4), we can easily prove (7.1).

For example, we notice that

$$
\frac{1}{2}+\frac{1}{6}+\frac{1}{12}+\frac{1}{20}+\frac{1}{30}+\frac{1}{42}+\frac{1}{56}+\cdots=\sum_{m=1}^{\infty} \frac{1}{m(m+1)}=1
$$

To prove (7.2), we use the recurrence relation for entries of Leibniz's triangle in equation (5.3) repeatedly. For example from Figure 5, we find that $\frac{1}{12}=\frac{1}{6}-\frac{1}{12}, \frac{1}{30}=\frac{1}{12}-\frac{1}{20}, \frac{1}{60}=\frac{1}{20}-\frac{1}{30}$

$\frac{1}{105}=\frac{1}{30}-\frac{1}{42}, \frac{1}{168}=\frac{1}{42}-\frac{1}{56}, \cdots$ Continuing this way repeatedly we find that

$$
\begin{aligned}
& \frac{1}{12}+\frac{1}{30}+\frac{1}{60}+\frac{1}{105}+\frac{1}{168}+\cdots \\
& =\left(\frac{1}{6}-\frac{1}{12}\right)+\left(\frac{1}{12}-\frac{1}{20}\right)+\left(\frac{1}{20}-\frac{1}{30}\right)+ \\
& +\left(\frac{1}{30}-\frac{1}{42}\right)+\left(\frac{1}{42}-\frac{1}{56}\right)+\cdots=\frac{1}{6}
\end{aligned}
$$

We thus see upon applying the recurrence relation property repeatedly, only the first term in the right hand side remains while all other terms cancel out mutually. This kind of summation is often referred as Telescopic Summation. Using the same principle we can select any infinite rectangular strip of numbers in the Leibniz's triangle whose sum would be a number just located above at the north-west corner of the strip. I refer this property as Inverted Infinite Hockey Stick Property. I now provide the general proof of this property. 


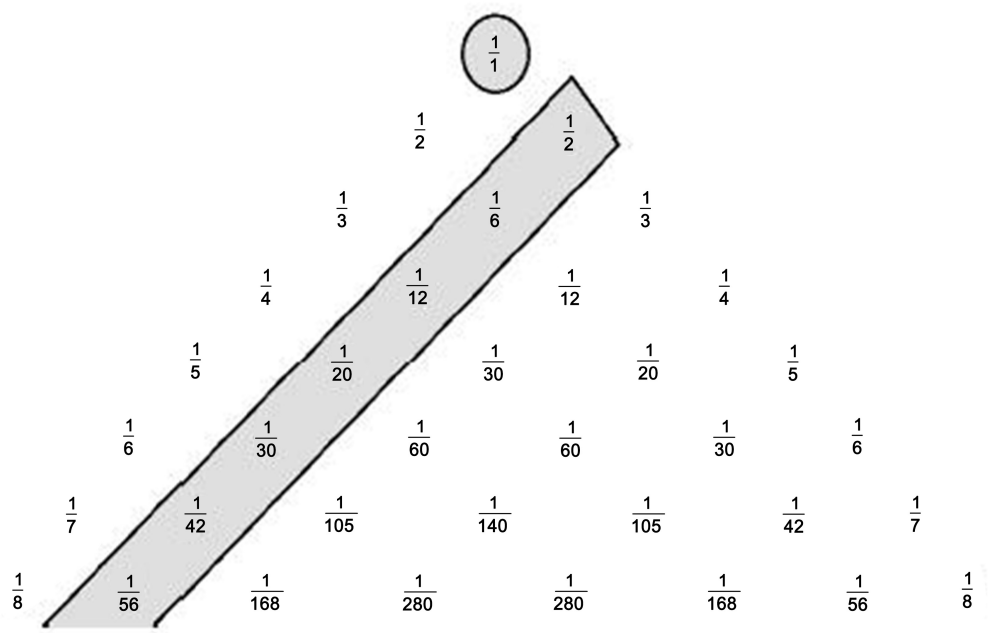

Figure 4. Illustration of Inverted Infinite Hockey Stick Property

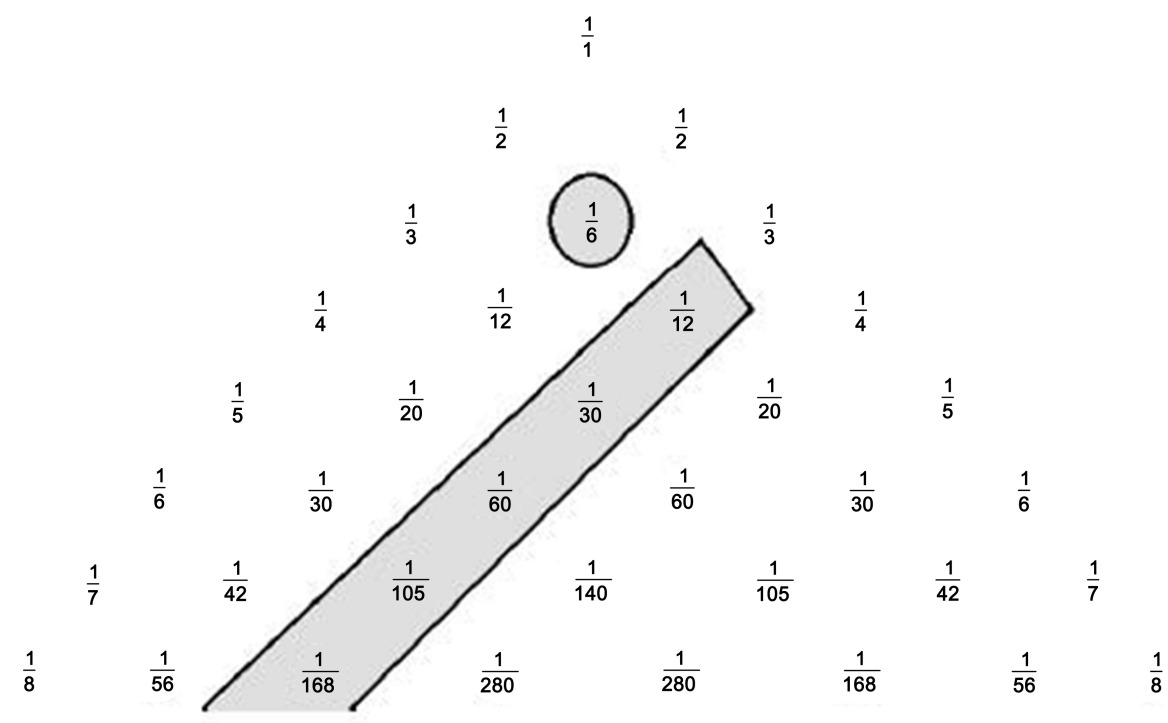

Figure 5. Illustration of Inverted Infinite Hockey Stick Property

\subsection{Inverted Infinite Hockey Stick Theorem}

If $m, n, r$ are whole numbers then

$$
\sum_{m=r}^{\infty} L_{m, n}=L_{r, n-1}
$$

Proof: Using (4.1), we have

$$
\begin{aligned}
& \sum_{m=r}^{\infty} L_{m, n}=\sum_{m=r}^{\infty} \int_{0}^{1} x^{m}(1-x)^{n} d x=\int_{0}^{1}\left(\sum_{m=r}^{\infty} x^{m}\right)(1-x)^{n} d x \\
& \sum_{m=r}^{\infty} L_{m, n}=\int_{0}^{1} x^{r}(1-x)^{n-1} d x=L_{r, n-1} . \text { This proves }(7.3)
\end{aligned}
$$

and completes the proof.

As an illustration, we find that for $r=1, n=2$ from (7.3), we get

$$
\sum_{m=1}^{\infty} L_{m, 2}=\frac{1}{12}+\frac{1}{30}+\frac{1}{60}+\frac{1}{105}+\frac{1}{168}+\cdots=L_{1,1}=\frac{1}{6}
$$

\section{Infinite Triangle Sum Property}

In Pascal's Triangle, we notice a Rhombus Shape as displayed in Figure 6.

As shown in Figure 6, if we add all the numbers inside the rhombus their sum would be $64=84-20$, which is the difference of the numbers located just vertically below the rhombus shown in circle and to the right of the number 10. This property of rhombus shape sum holds true for any rhombus taken in Pascal's triangle. Is there a similar property that can be observed in Leibniz's triangle? Figure 7 answers this question.

From Figure 7, we notice that if we add all the numbers inside the triangle, we get their sum as $\frac{1}{4}$ a number located just above the vertex of the infinite triangle. Like Pascal's triangle, this infinite triangle sum property also holds true for any triangle considered in Leibniz's triangle. I will now formally prove this fact. 


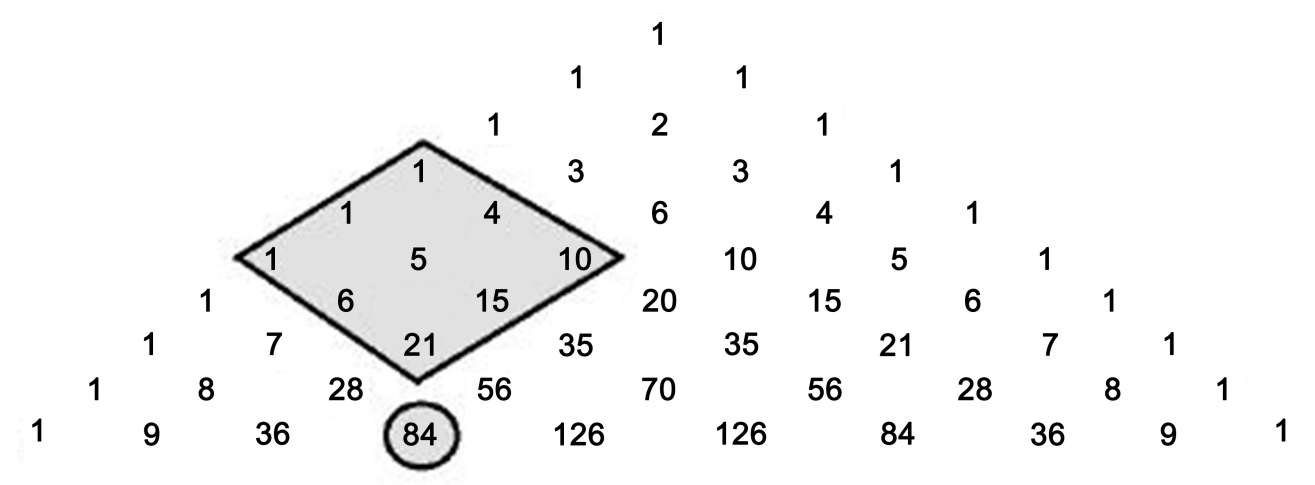

Figure 6. Rhombus Shape Sum Property in Pascal's Triangle

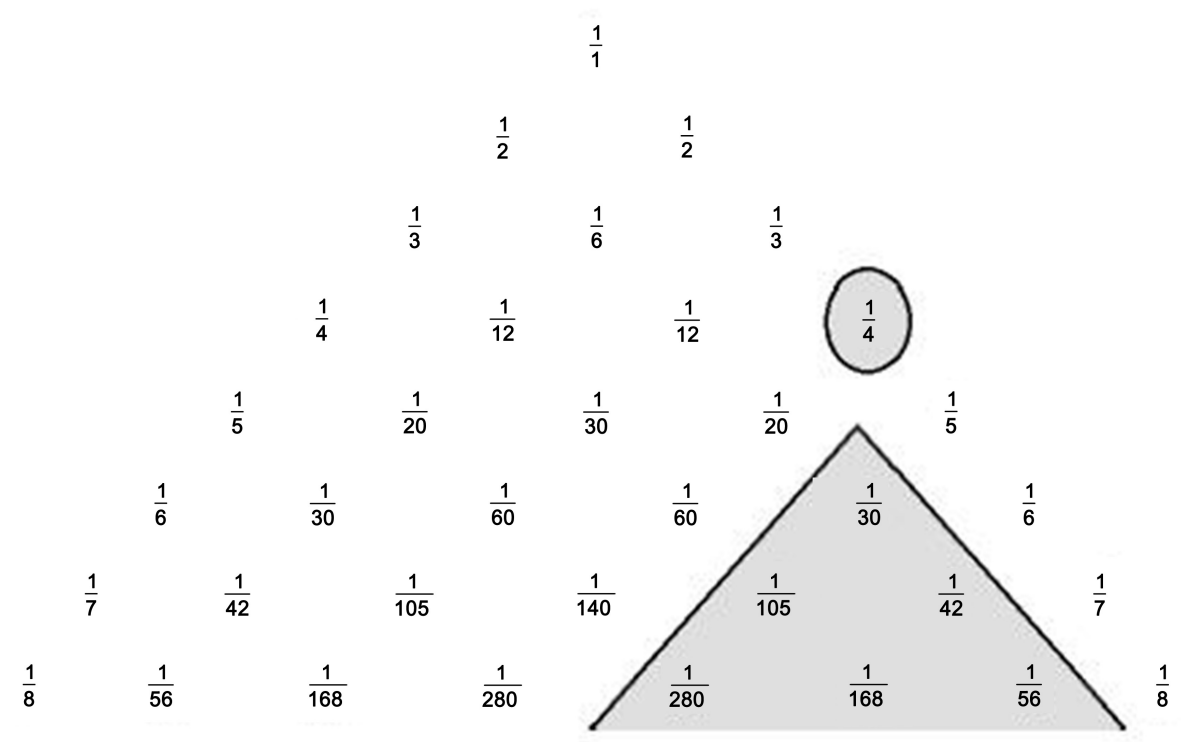

Figure 7. Infinite triangle sum property in Leibniz's triangle

\section{Infinite Triangle Sum Theorem}

If $m, n, r, k$ are whole numbers then

$$
\sum_{m=k}^{\infty} \sum_{n=r}^{\infty} L_{m, n}=L_{k-1, r-1}
$$

Proof: First, we note that $L_{k, r}$ is the topmost number in our infinite triangle. Also, we note from Figure 3 that $L_{k-1, r-1}$ is the number located just vertically above the vertex of the infinite triangle. We now use Inverted Infinite Hockey Stick Identity obtained in (7.3) twice to prove this theorem. First using (5.1), we get

$$
\begin{aligned}
& \sum_{m=k}^{\infty} \sum_{n=r}^{\infty} L_{m, n}=\sum_{m=k}^{\infty}\left(\sum_{n=r}^{\infty} L_{n, m}\right) \\
& =\sum_{m=k}^{\infty} L_{r, m-1}=\sum_{m=k}^{\infty} L_{m-1, r}=L_{k-1, r-1}
\end{aligned}
$$

This proves (8.1) and completes the proof.

I provide two illustrations explaining (8.1). First, considering Figure 7, we find that according to Figure 3, we have $k=1, r=4$. Note that the topmost entry of the infinite triangle of Figure 7 is $L_{1,4}=\frac{1}{30}$. Also note that the number in the circle above the vertex is $L_{0,3}=\frac{1}{4}$.

Hence the sum of all the numbers in the infinite triangle according to (8.1) would be $\sum_{m=1}^{\infty} \sum_{n=4}^{\infty} L_{m, n}=L_{0,3}=\frac{1}{4}$ as expected.

Similarly, the sum of all the numbers in the infinite triangle shown in Figure 8 below can be obtained by taking $k=1, r=1$.

Using (8.1), we have $\sum_{m=1}^{\infty} \sum_{n=1}^{\infty} L_{m, n}=L_{0,0}=1$, a number located above the vertex of the infinite triangle shown in the circle. 


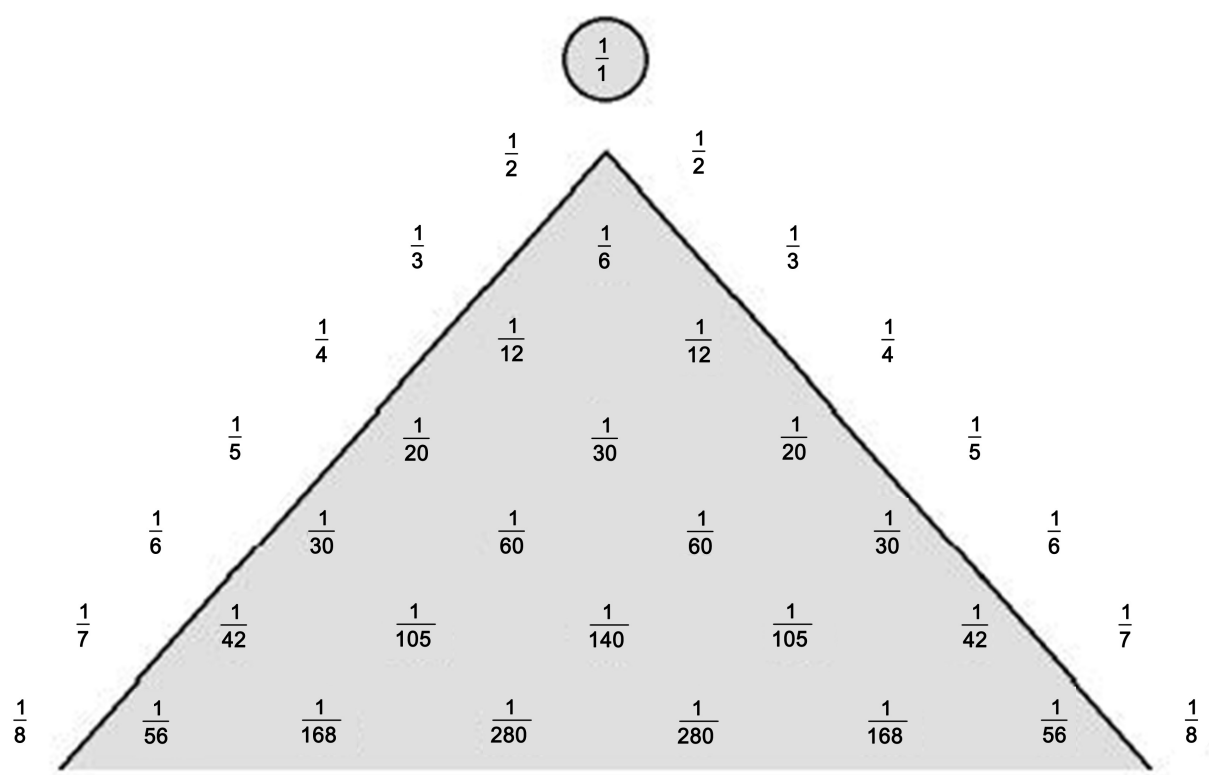

Figure 8. Infinite triangle sum property in Leibniz's triangle

\section{Conclusions}

Usually, the entries of Leibniz's triangle would either be identified through Recursion or obtained from Pascal's triangle as explained in section 3. But in this paper, I identified the entries of Leibniz's triangle as Beta Integral values which is not done before in any paper. I proved that the Beta Integral assumption is exactly same as that of entries obtained through Pascal's triangle in section 4 through equation (4.4). This equivalence made me establish so many identities in subsequent sections, that too very elegantly.

In section 5, I have proved five results in which the first four are commonly known, but the proofs are given with respect to integrals, which were not done before. The fifth result of section 5, namely sum of alternating terms of entries of Leibniz's triangle is not so well known, again proved easily by using integrals. The six results proved in section 6 are all relatively new and not much known in the literature about them. Hence, they are completely new results of this paper. In particular the proof of sum of all centralized Leibniz's triangle numbers is convergent provides a new dimension to existing properties.

In section 7, I had proved Infinite Hockey Stick Theorem, which is done so easily. As far as I knew there is no such easy way of proving as I did for that theorem. Further the Infinite Hockey Stick Theorem provides us a way of constructing gallery of several infinite series with unit fractions whose sum can be readily obtained by the equation (7.3). Similarly, in section 8, I had proved Infinite triangle sum property whose proof like previous one was done in a single line. Illustrations and Figures were provided for better understanding wherever necessary. Figures 4,5,6,7,8 were incorporated from [11].

This paper, thus contain almost all the known properties as well as unknown properties of the not so well known Leibniz's triangle. Most importantly by observing that the entries are Beta Integral values, I could prove almost every result in this paper quite beautifully and in short. This proves that by thinking little differently we can see deeper truths in a more elegant way.

\section{REFERENCES}

[1] Ivan D. Stones, "The harmonic triangle: Opportunities for pattern identification and generalization", The Mathematics Teacher, vol. 76, no. 5, pp. 350 - 354, May 1983, https://www.jstor.org/stable/27963528

[2] Peter Hilton and Jean Pedersen, "Looking into Pascal's triangle: Combinatorics, arithmetic, and geometry", Mathematics Magazine, vol. 60, no. 5, pp. 305 - 316, December 1987, https://doi.org/10.1080/0025570X.1987.11 977330

[3] Thomas Koshy,“Leibniz's Barmonic Triangle”, Triangular Arrays with Applications, Oxford University Press, New York, 2011.

[4] MarjorieBickNell-Johnson,"Diagonal sums in the harmonic triangle", Fibonacci Quarterly, vol. 19, no. 3, pp. 196 - 199, August 1981, https://www.fq.math.ca/Scanned/19-3/bicknel 1.pdf

[5] ANTOGNAZZA, Maria Rosa, "Leibniz: An Intellectual Biography, Cambridge”, Cambridge University Press, 2009.

[6] GeorgePolya, "Mathematical Discovery - On Understanding, Learning and Teaching Problem Solving", Wiley, 1981.

[7] Belaval, Yvon and Look, Brandon C.. "Gottfried Wilhelm Leibniz", Encyclopedia Britannica, https://www.britannica. com/biography/Gottfried-Wilhelm-Leibniz.

[8] Jerphagnon, Lucien and Orcibal, Jean. "Blaise Pascal", 
Encyclopedia Britannica, https://www.britannica.com/biogr aphy/Blaise-Pascal.

[9] S.C. Althoen and C.B. Lacampagne, "Tetrahedral numbers as sums of square numbers", Mathematics Magazine, pp. 104 108, 1991, https://doi.org/10.1080/0025570X.1991.1197758 6
[10] Alan Tucker, “Applied Combinatorics", Wiley $\left(6^{\text {th }}\right.$ Edition, USA, 2012

[11] James, Lacey Taylor, "Analogues between Leibniz's Harmonic Triangle and Pascal's Arithmetic Triangle" (2019). Electronic Theses, Projects, and Dissertations, 835, https://scholarworks.lib.csusb.edu/etd/835 